\title{
Optimizing drug therapy in patients with advanced dementia: A patient-centered approach
}

\author{
N. Molist Brunet ${ }^{\mathrm{a}, *}$, D. Sevilla-Sánchez ${ }^{\mathrm{b}}$, J. Amblàs Novellas ${ }^{\mathrm{a}}$, C. Codina Jané $^{\mathrm{c}}$, \\ X. Gómez-Batiste ${ }^{\mathrm{d}}$, J. McIntosh ${ }^{\mathrm{e}}$, J. Espaulella Panicot ${ }^{\mathrm{a}}$ \\ ${ }^{a}$ Hospital de la Santa Creu, Consorci Hospitalari de Vic, Vic, Spain \\ ${ }^{\mathrm{b}}$ Hospital de la Santa Creu, Hospital General de Vic, consorci Hospitalari de Vic, Vic, Spain \\ ${ }^{\mathrm{c}}$ Hospital Clínic de Barcelona, Hospital General de Vic, Vic, Barcelona, Spain \\ d Palliative Care Cathedra, Universidad de Vic, Vic, Barcelona, Spain \\ e Hospital Clínic de Barcelona, Barcelona, Spain
}

\section{A R T I C L E I N F O}

\section{Article history:}

Received 3 July 2013

Accepted 24 October 2013

Available online 21 November 2013

\section{Keywords:}

Polypharmacy

Advanced dementia

End-of-life care

\begin{abstract}
A B S T R A C T
Background: Advanced dementia is a prevalent health problem in geriatric patients. These patients usually suffer from several chronic diseases, frequently leading to an end-of-life situation lasting months or years, generating complex and often inappropriate medication regimens.

Objectives: Describe the re-orientation of drug therapy in patients with advanced dementia utilizing a systematic medication review process.

Methods: This non-experimental pre-post analysis included all patients with advanced dementia admitted to acute geriatric unit (AGU) over one year. Medications were reviewed by a multidisciplinary team and together with the patient caregivers; new therapeutic objectives based on end-of-life care principles were established. Medications were classified as preventive, therapeutic, or symptomatic. The average number of medications per patient pre- and post-admission was compared.

Results: We included 73 patients (mean age 86.1 years, mean Barthel Index: 14.5/100). At admission, patients had a mean of 7.27 drugs compared to 4.82 at discharge $(66.85 \%$ reduction, $P<0.05)$. The main drugs withdrawn were cardiovascular and hematological (35.76\%). Drugs for prevention decreased by $66.85 \%$ (from 1.8 to $0.6, P<0.05$ ) and those for symptomatic care decreased by $17,52 \%$ (from 2.34 to 1.93 , $P<0.05)$.

Conclusion: Medication therapy plans in patients with advanced dementia often do not meet their therapeutic goals. The proposed methodology is a useful tool to assess therapeutic appropriateness.

(c) 2013 Elsevier Masson SAS and European Union Geriatric Medicine Society. All rights reserved.
\end{abstract}

\section{Introduction}

As the world population continues aging, there will be an increasing number of patients with advanced, complex chronic disease [1]. Many of these patients will deteriorate clinically, functionally, or cognitively, ultimately resulting in a situation of limited prognosis [2]. Advanced dementia is one such chronic condition, and is a problem of serious consequence with a prevalence as high as $80 \%$ in nursing home patients, making it a pressing concern for both patients and providers [3,4].

Multi-morbidity is common amongst patients with chronic conditions. This often leads to complex treatment regimens,

\footnotetext{
* Corresponding author. Acute Geriatric Units, Hospital General de Vic. C/ Francesc Pla el Vigatà, s/n, 08500 Vic, Barcelona, Spain. Tel.: +34 93889 1111, ext: 1503.

E-mail address: nmolist@gmail.com (N. Molist Brunet).
}

evidenced by the presence of polypharmacy (defined as five or more medications) [5] and by the heterogeneity of therapeutic goals, which often include preventive, therapeutic, and symptomatic objectives. When approaching end-of-life care, as in the case of advanced dementia, both the number of and the indication for the medication should be evaluated, with the priority placed on symptomatic versus prevention care.

Pharmaceutical care is well defined in the last days or hours of life, with the primary focus on symptomatic care and the alleviation of suffering [6-8]. In contrast, pharmacological care during the broader end-of-life time frame is less well defined and there are no specific guidelines, owing in part to limited clinical trial data in this population and the uncertain prognosis of these patients [6,9]. Although during this stage a primary goal is symptom management, this is not incompatible with other treatment objectives. Thus, a dynamic and systematic review of a patient's medication profile is called for, with the objective of 
adjusting therapy to meet the true needs of the patient. This often requires a re-orientation in regard to therapies for chronic conditions, including both initiating discontinuing, medications chronic medications.

Raising the withdrawal of chronic therapies constitutes a professional challenge for several reasons:

- it requires the identification of patients who are within the last months or year of life;

- it requires defining new drug therapy goals, evaluating the potential risks of discontinuing a specific medication [10].

Both of these are feasible in patients with advanced dementia, a disease well established as a terminal illness where the primary goals focus on symptomatic care [11]. Furthermore, removal of unnecessary drugs may be beneficial to the patient, as complex drug therapy and the resulting polypharmacy may increase the risk of adverse drug events [12-14].

Recently, published recommendations for end-of-life pharmacotherapy provide guidance on individualizing drug therapy regimens [15-17]. This requires re-thinking the prescription process, utilizing criteria specific to this patient population including:

- avoiding drug therapy where the primary goal is extending life;

- avoiding treatments for primary prevention (the time-to-benefit is longer than the patient's life expectancy);

- individualizing the use of secondary prevention (ensuring timeto-benefit is within the expected life span);

- reducing the number of medications per patient, moving from a state of polypharmacy to oligopharmacy (less than 5 medications per patient);

- defining treatment goals jointly with patients or caregiver;

- acknowledging that the process will be dynamic and will require continuous reassessment;

- involving multiple health care professionals to create multidisciplinary care teams [15].

The objective of the study is to describe the re-orientation of drug therapy utilizing a systematic approach focusing on patient cantered goals in a group of patients with advanced dementia admitted to an acute geriatric unit (AGU).

\section{Methods}

We conducted a non-experimental pre-post evaluation of the standard medical management of patients admitted to the AGU in a secondary care regional hospital in Catalonia, Spain between May 2011 and April 2012. This evaluation includes patients with advanced dementia, defined as either having all three of the following: an inability to complete activities of daily living (Barthel index less than or equal to 30/100), incontinence and difficulty recognizing family members (Global Deterioration Scale greater than or equal to $6 \mathrm{~d}$ in case of Alzheimer type dementia). Patients who died during hospitalization were excluded.

\subsection{Developing medication therapy plan}

In order to create a new patient cantered drug therapy regimen, all patient medication profiles were systematically evaluated in a three-stage process by a multidisciplinary team consisting of two geriatricians and a clinical pharmacist (Fig. 1).

\subsubsection{Patient centered assessment}

We conducted an initial multidimensional geriatric assessment of clinical, functional, cognitive, and social indicators. The objective of this review was to employ a goal-oriented approach, taking into account the evolutionary state of the chronic disease, to guide care decisions [18]. If patients were not able to communicate for themselves, the values and preferences of caregivers were taken into account when establishing therapeutic goals. At the end of this review, new therapeutic goals were established, guided by the known criteria for recognizing key transitions at the end-of-life [19].

\subsubsection{Diagnosis centred assessment}

Patient health problems were listed together with the medications prescribed for each diagnosis. Each medication was then classified by its objective and placed into one of the following categories:

- preventive therapies (primary or secondary);

- therapeutic or curative treatments;

- or symptomatic therapy.

In this stage, we evaluated the applicability of clinical practice guidelines according to each patient's therapeutic goals. Although all medications were reviewed, special attention was paid to those previously identified in the literature as having a high potential for discontinuation at the end-of-life, such as those used for primary prevention. (Table 1) [15-17]. This allowed us to make prescribing decisions based on both the therapeutic objective of the medication and the previously established therapeutic goals of the patient.

\subsubsection{Medication centred assessment}

We also assessed the medication profile for drug-related problems (Fig. 1).

Based on this process, we developed patient-specific therapeutic plans for each patient.

\subsection{Data collection and analysis}

Basic patient demographic information was collected, including age, sex, living arrangements (own home or residence), and discharge destination. The Barthel Index was used to measure functionality at admission and at discharge. The chief complaint, length of stay, and presence of delirium during hospitalization were also recorded. To assess the presence of polypharmacy, the number of medications pre- and post-admission were collected, along with the number of medication added or discontinued during the hospitalization.

Finally, we performed a statistical analysis consisting of calculating the differences in the number of medications at admission and discharge using the non-parametric Wilcoxon signed-rank test (SPSS software 15.0) with a significance of $P<0.05$. Non-normality of distribution was assessed by goodnessof-fit test of Kolmogorov-Smirnov.

\section{Results}

\subsection{Patient demographics}

During the study year, a total of 934 patients were admitted to the AGU, of which $73(7.81 \%)$ met the inclusion criteria.

Of those included in the study, $79.45 \%$ were women and the mean age was 86.1 years (SD: 5.73 , range $72-100$ ). The majority of patients (58.9\%) were institutionalized (from nursing homes) and the remainder were living at home. The mean Barthel Index of the study population was $14.5 / 100$.

The main admission diagnoses were: trauma $35.61 \%$ (fracture of the femur $84.52 \%$ ), infection $36.98 \%$ (respiratory infections $44.34 \%$ 


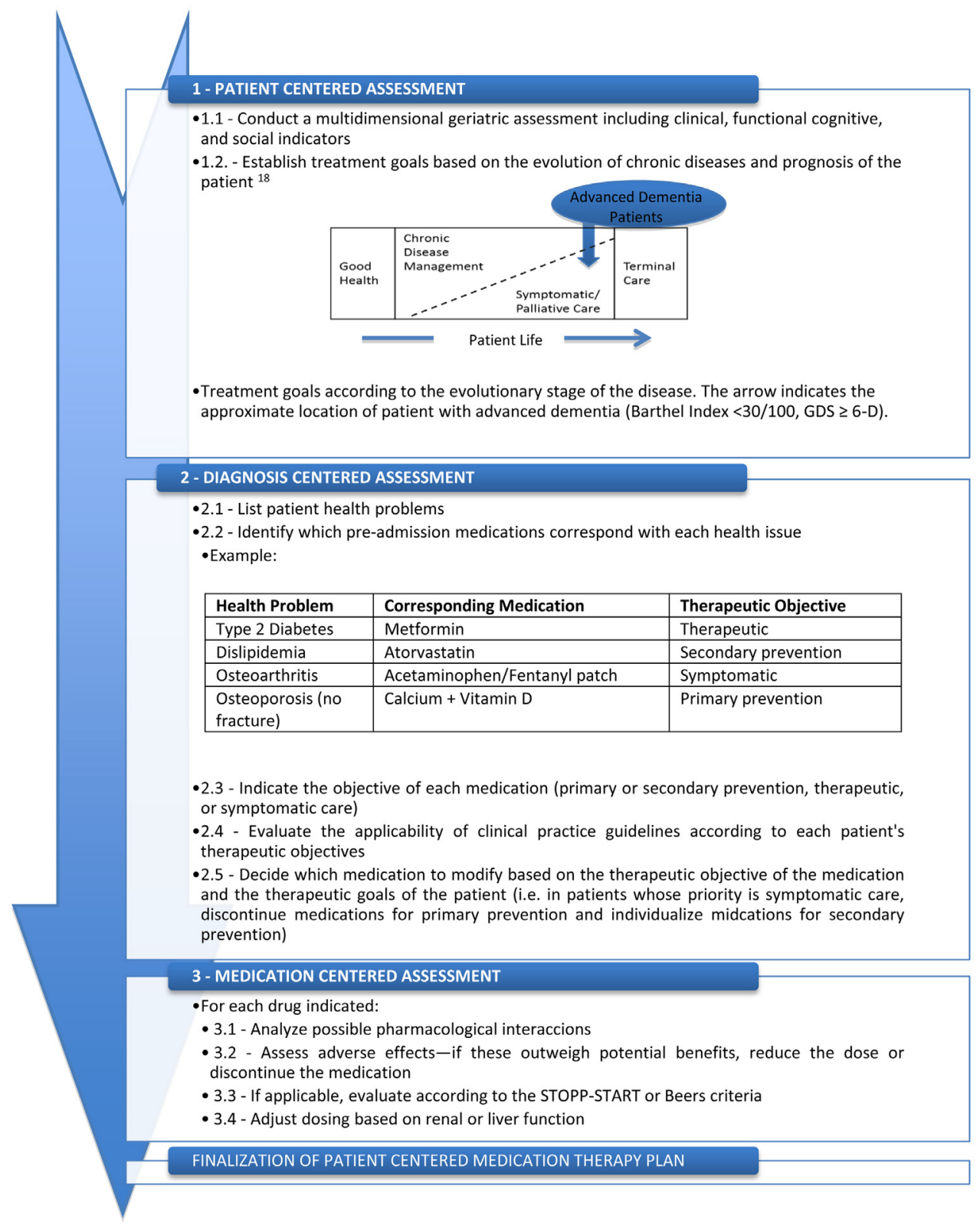

Fig. 1. Methodology for development of patient centered medication plan.

and urinary tract infections 33.26\%), and cardiovascular disease $20.54 \%$ (worsening of heart failure, acute coronary syndrome and cardiac dysrhythmia, etc.). The average stay was 4.89 days (global average during the same period for the entire unit was 6.2 days). Almost half $(43.80) \%$ of the patients had acute delirium during hospitalization. At discharge, $57.5 \%$ of the patients were referred to their original place of residence and the remaining $42.5 \%$ were discharged to an intermediate care hospital.

\subsection{Medication profiles}

Patients had an average of 7.27 medications prior to hospitalization, and $82.2 \%$ met the criteria for polypharmacy. Of these medications, $24.80 \%$ (average of 1.81 per patient) were for prevention, of which $76.2 \%$ were for primary prevention (average of 1.38 per patient) and $23.8 \%$ were for secondary prevention (average of 0.43 per patient). Medications for therapeutic purposes represented $42.91 \%$ of all medications (mean of 3.12 per patient), and those for symptomatic care represented $32.2 \%$ (mean of 2.34 per patient) (Table 2 ).
The average number of medications per person at discharge decreased by 2.45 per person (66.30\%) to 4.8 , excluding temporary medications initiated to address the chief complaint (Table 2). Decreases were seen in all groups (preventive, therapeutic and symptomatic) although the most commonly discontinued medications were for prevention. Two-thirds (66.85\%) of the preventive medicines were discontinued due to lack of evidence in an elderly patient population, with the vast majority (78.50\%) indicated for primary prevention. More than a quarter (27.24\%) of drugs designated for treatment were discontinued due to lack of a clear indication, and drugs for symptomatic control were also decreased by $17.52 \%$.

The most common systems affected by medication discontinuations were the cardiovascular and blood system, accounting for $35.70 \%$ of all discontinued medications, followed by drugs for the nervous system (19.56\%), drugs related to the metabolism or nutrition (16.77\%), and musculoskeletal system (14.52\%) (Table 3 ).

During the admission, new medications were started, of which $54.30 \%$ were to treat the chief complaint and were of limited duration. The most frequently added new medications were: 
Table 1

Therapeutic groups, based on anatomical therapeutic chemical (ATC) classification system, evaluated in patients at the end-of-life.

\begin{tabular}{|c|c|}
\hline Anatomical group & Therapeutic class \\
\hline \multicolumn{2}{|l|}{ A: Alimentary tract and metabolism } \\
\hline \multirow[t]{2}{*}{ Antidiabetics } & Metformin \\
\hline & Thiazolidinediones \\
\hline \multirow[t]{2}{*}{ Anti-ulcer (Gastroprotectants) } & Proton Pump Inhibitors \\
\hline & Antihistamines \\
\hline \multicolumn{2}{|l|}{ B: Blood and blood forming organs } \\
\hline Antiplatelet and anticoagulant & Acetylsalicylic acid and clopidogrel \\
\hline \multirow[t]{3}{*}{ Anti-anemics } & Acenocoumarol, warfarina, dabigatran, rivaroxaban \\
\hline & Iron \\
\hline & Vitamin $\mathrm{B}_{12}$; folic acid \\
\hline \multirow[t]{2}{*}{ Lipid modifying agents } & Statins \\
\hline & Fibrates \\
\hline \multicolumn{2}{|l|}{ C: Cardiovascular } \\
\hline \multirow[t]{2}{*}{ Antihypertensives } & Angiotensin converting enzyme inhibitors for prevention of nephropathy \\
\hline & Angiotensin II receptor antagonists for prevention of nephropathy \\
\hline \multicolumn{2}{|r|}{ 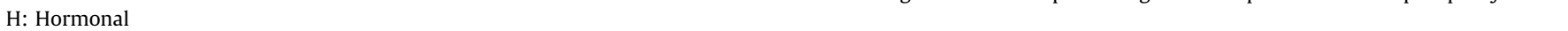 } \\
\hline \multirow[t]{4}{*}{ Drugs for bone-disease } & Calcium and vitamin D supplements \\
\hline & Bisphonophonates \\
\hline & Teriparatide \\
\hline & Estrogen \\
\hline \multicolumn{2}{|l|}{$\mathrm{J}:$ Anti-infectives } \\
\hline Bacterial infections & Prophylactic antibiotics Antibióticos profilácticos \\
\hline \multicolumn{2}{|c|}{ L: Antineoplastics and immunomodulating agents } \\
\hline Cytotoxics & Cytotoxic and chemotherapeutic agents \\
\hline Vaccines & Preventive vaccines \\
\hline \multicolumn{2}{|l|}{ M: Musculoskeletal } \\
\hline Anti-inflammatory & Non-steroidal anti-inflamatories \\
\hline Antiarthritics & Cytotoxic and biologic therapy (monoclonal antibodies) \\
\hline \multicolumn{2}{|l|}{$\mathrm{N}$ : Nervous system } \\
\hline \multirow[t]{2}{*}{ Anti-dementia drugs } & Acetylcholinesterase inhibitors \\
\hline & Memantine \\
\hline Anti-Parkinson & Anticholinergics to treat extrapyramidal effects of neuroleptics \\
\hline Antidepressants & Tricyclic antidepressants \\
\hline Anxiolytics and hypnotics & Benzodiazapines with long half-life \\
\hline \multicolumn{2}{|l|}{ R: Respiratory system } \\
\hline Antiasthmatics & Theophyline \\
\hline
\end{tabular}

antibiotics, (20.43\%), low-molecular weight heparin (18.28\%), corticosteroids (8.60\%) and gastroprotectants (6.99\%). Although all patients were receiving end-of-life care, some medications were also added for therapeutic purposes, representing $20.43 \%$ of all new medications. Digoxin for atrial fibrillation and antihypertensives were the most commonly added. Finally, $24.73 \%$ of new drugs were to improve symptom control, such as analgesics NSAIDs (26.90\%), laxatives (18.40\%) and neuroleptics (16.10\%).

Although the total number of medications in each three groups decreased, because significantly more medications for prevention were discontinued compared to therapeutic or symptomatic care, the later two represented a larger proportion of the overall medication profile at discharge (Table 2). For example, at admission, therapeutic medications represented $42.90 \%$ of all drugs, but this proportion rose to $47.10 \%$ at discharge. Similarly, symptomatic medications went from $32.20 \%$ to $40.20 \%$ while preventive medications fell from $24.80 \%$ to only $12.65 \%$.

\section{Discussion}

The optimization of medication therapy in frail patients at the end-of-life remains a challenge to the current healthcare system. This study demonstrates that patients with advanced dementia are often maintained on drug therapy for chronic conditions that fail to take into account the patient's global condition. This is reflected quantitatively by the fact that polypharmacy is common amongst this population and qualitatively, in that the therapy was not targeted to the real needs of the patient. We propose a systematic methodology that personalizes medication therapy, which we applied to patients with advanced dementia. In this subgroup of patients, we significantly decreased the number of prescribed medications, from an average of 7.3 chronic medications per patient at admission to 4.8 at discharge. This reduction was primarily seen amongst medications for primary prevention.

Table 2

Preventive, therapeutic and symptomatic medications affected by proposed changes in therapeutic goals.

\begin{tabular}{|c|c|c|c|c|c|}
\hline & \multicolumn{4}{|c|}{ Average number of medications per patient } & \multirow[b]{2}{*}{$P$} \\
\hline & Admission & $\begin{array}{l}\text { Discharge (including short-term } \\
\text { and long-term medications) }\end{array}$ & $\begin{array}{l}\text { Discharge (excluding short-term, } \\
\text { admission-related medications) }\end{array}$ & $\begin{array}{l}\text { Difference between } \\
\text { admission and discharge }\end{array}$ & \\
\hline Preventive therapies & $1.81(24.8 \%)$ & $1.44(19.61 \%)$ & $0.60(12.65 \%)$ & $-1.21(-66.85 \%)$ & $<0.05$ \\
\hline Primary & $1.38(18.9 \%)$ & $1.30(17.71 \%)$ & $0.43(8.92 \%)$ & $-0.95(-68.84 \%)$ & $<0.05$ \\
\hline Secondary & $0.43(5.9 \%)$ & $0.14(1.90 \%)$ & $0.17(3.52 \%)$ & $-0.26(-60.46 \%)$ & $<0.05$ \\
\hline Therapeutic treatments & $3.12(42.91 \%)$ & $3.41(46.45 \%)$ & $2.27(47.1 \%)$ & $-0.85(-27.24 \%)$ & $<0.05$ \\
\hline Symptomatic treatment & $2.34(32.2 \%)$ & $2.49(33.92 \%)$ & $1.93(40.24 \%)$ & $-0.41(-17.52 \%)$ & $<0.05$ \\
\hline Total & 7.27 & 7.34 & 4.8 & $-2.45(-66.3 \%)$ & $<0.05$ \\
\hline
\end{tabular}


Table 3

Type of medications changed or discontinued by organ system and drug class.

\begin{tabular}{ll}
\hline Organ system & Drug class \\
\hline $\begin{array}{c}\text { Cardiovascular and } \\
\text { hematologic (35.76\%) }\end{array}$ & Antiplatlets: $13.41 \%$ \\
& Antihypertensives: $9.50 \%$ \\
& Hipolypidemics: $5.59 \%$ \\
& Anticoagulants: $2.23 \%$ \\
& Other cardiovascular: $5.03 \%$ \\
Antidepressants: $9.50 \%$ \\
Nervous system (19.56\%) & Benzodiazapines and neuroleptics: $8.38 \%$ \\
& Dementia therapies: $1.68 \%$ \\
Alimentary tract and & Nutritional supplements: $8.94 \%$ \\
metabolism (16.77\%) & Gastroprotectants: $6.15 \%$ \\
& Antidiabetics: $1.68 \%$ \\
Musculoskeletal (14.52\%) & Antiresorptive: $7.82 \%$ \\
& Analgesics (NSAIDs): $6.70 \%$ \\
Respiratory (1.68\%) & Anti-asthmatics \\
Genitourinary (0.56\%) & Antispasmodics and anticholinergics \\
Other (11.15\%) & Eyedrops \\
& Low value intrinsic medications: brain \\
& vasodilators and nootropics drugs; \\
& chondroprotectors \\
\hline
\end{tabular}

These results are consistent with other studies in frail elderly patients in other healthcare settings [20-22]. A program to address polypharmacy in elderly community dwelling patients resulted in an average decrease of 4.4 medication per patient [21] whereas an intervention in a geriatric specialty hospital decreased medications by $47 \%$ (2.8) per patient [20]. Importantly, these previously published studies did not report an increase in adverse events, and families reported an increase in the quality of life, functionality, and cognitive status.

Although it was not the primary objective of this study, our results confirm that patients with advanced dementia present with high levels of dependency, as more than half of the patients in this study were living in an institutional setting. We also confirm that in this population, infection and trauma are the most frequent reasons for hospitalization, followed by the worsening of other chronic conditions. Furthermore, we observed frequent transitions between care levels with nearly half of discharged patients not returning to the original living situation and with many of these going to an intermediate care unit. This highlights the need for clear communication between hospitalists, primary care providers, patients, and healthcare providers in intermediate care facilities to prevent medication errors and ensure continuity of care [23].

Studies in patients with advanced dementia have typically emphasized the natural history of the disease and the resulting problems, but few have resulted in interventions that improve pharmacological outcomes [10]. Particularly in the area of drug therapy, interventions have primarily focused on anticholinesterases and neuroleptics [24], but interventions addressing the overall patient status are lacking. In this study, we describe a methodology to personalize medication therapy in patients with advanced dementia, resulting in medication therapy plans that take into account the global status of the patient. This type of patient-centred drug therapy intervention is the basis of patientcentred care [18], and is applicable to other patients at the end-oflife, including those with extremely frailty or with advanced stage organ disease or cancer [25]. For example, our proposed systematic review of drug therapy can be used in parallel with existing tools designed to identify potentially inappropriate medications in the elderly, such the Beers criteria or STOPP/START [26,27], which may not always take into account the needs of an individual patient.

This work reflects the process developed by our multidisciplinary team of geriatricians and a clinical pharmacist, and represents a continuous evaluation of the drug therapy profile.
The basis of this process is establishing appropriate therapeutic goals at the end-of-life, and then realigning drug therapy to meet these goals.

One of the limitations of the current study is the lack of data on patient outcomes. Assessing the health and quality of life, adverse events due to medication withdrawal, and the economic impact of our intervention would have strengthened the results. Future research should address these questions.

In conclusion, patients with advanced dementia often receive inadequate drug therapy with many medications prescribed for primary prevention, and the majority of patients meeting the criteria for polypharmacy. Consequently, these patients could benefit from the personalization of their medication therapy from a multidisciplinary team, leading to better agreement between clinical objective that prioritize symptomatic control and the patient's medication profile.

\section{Disclosure of interest}

The authors declare that they have no conflicts of interest concerning this article.

\section{Acknowledgments}

We thank to Pere Roura for technical help with statistical analysis.

\section{References}

[1] World Health Organization National Institute on Aging, National Institute of Health. Global Health and Aging; 2011, http://www.who.int/ageing/publications/global_health/en/index.html. Accessed May 2013.

[2] Thomas K, et al. Prognostic Indicator Guidance. 4th Ed., The Gold Standards Framework Centre in End of Life Care 2011, 13, 4th Ed. 2012. http://www.goldstandardsframework.org.uk/LibraryofResourcesPresentationsandLinks.html. Accessed December.

[3] Mitchell SL, Kiely DK, Hamel MB. Dying with advanced dementia in the nursing home. Arch Intern Med 2004;164(3):321-6.

[4] Lopez Mongil R, Lopez Trigo JA, Castrodeza Sanz FJ, Tamames Gomez S, Leon Colombo T, Grupo de Trabajo de Atencion Sanitaria en Residencias de Ancianos de la Sociedad Espanola de Geriatria y G. [Prevalence of dementia in institutionalized patients. The RESYDEM study]. Rev Esp Geriatr Gerontol 2009:44(1):5-11.

[5] Gnjidic D, Hilmer SN, Blyth FM, et al. Polypharmacy cutoff and outcomes: five or more medicines were used to identify community-dwelling older men at risk of different adverse outcomes. J Clin Epidemiol 2012;65(9):989-95.

[6] Hall S. World Health Organization Regional Office for Europe. In: Palliative care for older people: better practices. Copenhagen, Denmark: World Health Organization, Regional Office for Europe; 2011.

[7] Maltoni M, Scarpi E, Rosati M, et al. Palliative sedation in end-of-life care and survival: a systematic review. J Clin Oncol 2012;30(12):1378-83.

[8] Qaseem A, Snow V, Shekelle P, et al. Evidence-based interventions to improve the palliative care of pain, dyspnea, and depression at the end of life: a clinical practice guideline from the American College of Physicians. Ann Intern Med 2008;148(2):141-6.

[9] Crome P, Lally F, Cherubini A, et al. Exclusion of older people from clinical trials: professional views from nine European countries participating in the PREDICT study. Drugs Aging 2011;28(8):667-77.

[10] Holmes HM, Hayley DC, Alexander GC, Sachs GA. Reconsidering medication appropriateness for patients late in life. Arch Intern Med 2006;166(6):605-9.

[11] Mitchell SL, Black BS, Ersek M, et al. Advanced dementia: state of the art and priorities for the next decade. Ann Intern Med 2012;156(1 Pt 1):45-51.

[12] Le Couteur D, Banks E, Gnjidic DAM. Deprescribing. Aust Prescr 2011;34: $182-5$.

[13] Marcum ZA, Amuan ME, Hanlon JT, et al. Prevalence of unplanned hospitalizations caused by adverse drug reactions in older veterans. J Am Geriatr Soc 2012:60(1):34-41.

[14] Hartholt KA, van der Velde N, Looman CW, et al. Adverse drug reactions related hospital admissions in persons aged 60 years and over. The Netherlands, 1981-2007: less rapid increase, different drugs. PLoS One 2010;5(11):e13977.

[15] O'Mahony D, O'Connor MN. Pharmacotherapy at the end-of-life. Age Ageing $2011 ; 40(4): 419-22$

[16] Schiff GD, Galanter WL, Duhig J, Lodolce AE, Koronkowski MJ, Lambert BL. Principles of conservative prescribing. Arch Intern Med 2011;171(16):143340 .

[17] Cruz-Jentoft AJ, Boland B, Rexach L. Drug therapy optimization at the end of life. Drugs Aging 2012;29(6):511-21. 
[18] Reuben DB, Tinetti ME. Goal-oriented patient care - an alternative health outcomes paradigm. Engl N J Med 2012;366(9):777-9.

[19] Boyd K, Murray SA. Recognising and managing key transitions in end of life care. BMJ 2010;341:c4863.

[20] Garfinkel D, Zur-Gil S, Ben-Israel J. The war against polypharmacy: a new costeffective geriatric-palliative approach for improving drug therapy in disabled elderly people. Isr Med Assoc J 2007;9(6):430-4.

[21] Garfinkel D, Mangin D. Feasibility study of a systematic approach for discontinuation of multiple medications in older adults: addressing polypharmacy. Arch Intern Med 2010;170(18):1648-54.

[22] Beer C, loh PI, Peng YG, Potter KAM. A pilot randomized controlled trial of deprescribing. Ther Adv Drug Safe 2011;2:37-43.

[23] Coleman EA, Berenson RA. Lost in transition: challenges and opportunities for improving the quality of transitional care. Ann Intern Med 2004;141(7):5336.
[24] Martin G, Sabbagh M. Palliative Care for Advanced Alzheimer's and Dementia. New York: Springer Publishing Company; 2010, http://JHU.eblib.com/patron/ FullRecord.aspx?p=667355.

[25] Gómez-Batiste X, Martínez-Muñoz M, Blay C, Amblàs J, Vila L, et al. CXe. Identifyung patients with chronic conditions in need of palliative care in the general population: development of the NECPAL tool and preliminary prevalence rates in Catalonia. BMJ Support Palliat Care 2013. 10.1136/bmjspcare2012-000211.

[26] American Geriatrics Society Beers Criteria Update Expert P.. American Geriatrics Society updated Beers Criteria for potentially inappropriate medication use in older, adults. J Am Geriatr Soc 2012;60(4):616-31.

[27] Gallagher P, Ryan C, Byrne S, Kennedy J, O’Mahony D. STOPP (Screening Tool of Older Person's Prescriptions) and START (Screening Tool to Alert doctors to Right Treatment). Consensus validation. Int J Clin Pharmacol Ther 2008;46(2):72-83. 